\title{
Robust dynamic positioning of surface vessels via multiple unidirectional tugboats
}

\author{
Baris Bidikli ${ }^{a}$, Enver Tatlicioglu ${ }^{\mathrm{a}, *}$, Erkan Zergeroglu ${ }^{\mathrm{b}}$ \\ ${ }^{a}$ Department of Electrical E Electronics Engineering, Izmir Institute of Technology, Izmir 35430, Turkey \\ ${ }^{\mathrm{b}}$ Department of Computer Engineering, Gebze Technical University, 41400 Gebze, Kocaeli, Turkey
}

\section{A R T I C L E I N F O}

\section{Article history:}

Received 30 April 2015

Accepted 30 December 2015

Available online 19 January 2016

Keywords:

Surface vessel

Positioning control

Tugboats

Robust control

\begin{abstract}
A B S T R A C T
In this paper, the problem of accurate positioning of an unactuated surface vessel by using multiple unidirectional tugboats is investigated. Specifically a robust controller that ensures asymptotic position tracking is designed. The control design procedure is implemented in two steps: Initially by locating opposing tugboats to specific configurations, the overall problem is transformed into a second order system with an uncertain non-symmetric input gain matrix. Then via a matrix decomposition, a novel robust controller methodology is proposed. The stability of the overall system is ensured via rigorous stability analysis where asymptotic position tracking is ensured. Numerical simulation results are presented to demonstrate the efficiency of the proposed controller.
\end{abstract}

(c) 2016 Elsevier Ltd. All rights reserved.

\section{Introduction}

Position control of large surface vessels like barges, offshore platforms and unactuated ships, throughout a narrow canal or in crowded harbors is an extremely delicate and important application as these large vessels usually are not designed, or not able to generate the necessary control effort to maneuver in these circumstances. Manipulation with multiple tugboats is a feasible solution for maneuvering these type of surface vessels. The motion objective is realized via a group of tugboats that are strategically positioned along the vessel's hull. When this operation is performed manually, due to the radio communication between all involved tugboats, the overall control performance is effected dramatically. Although, the communication performance is increased with advanced global positioning systems, control of these type of systems is still challenging due to possible problems that may arise in the communication system during the manipulation. As a result, positioning of unactuated surface vessels has attracted attention of automatic control researchers.

In the last decade, different types of automatic controller designs have been proposed for these type of applications. Recently, in Vlachos and Papadopoulos (2013), modeling of a novel triangle-shaped floating marine vessel was presented along with the design of a feedback linearization controller. The controller proposed required accurate model knowledge and achieved

\footnotetext{
* Corresponding author. Tel.: +90 232 7506536; fax: +90232 7506599 .

E-mail address: enver@iyte.edu.tr (E. Tatlicioglu).
}

ultimately bounded position tracking result. In Feemster et al. (2006), orientation tracking control of an unactuated vessel through the utilization of a swarm of vehicles operating in a decentralized fashion was achieved via a robust control strategy. In this design, the influence of other swarm vehicles was treated as a force disturbance acting on system dynamics. In Smith et al. (2007), an exact model knowledge position and orientation tracking controller was proposed for an unactuated surface vessel. Feemster and Esposito (2011) designed a tracking controller subject to control saturation due to the limitations of the tugboats where accurate knowledge of the dynamic model of the unactuated surface vessel was utilized in the control design. In Braganza et al. (2007), an adaptive position control strategy that does not require the location of the tugboats about the vessel hull was proposed. The adaptive controller proposed in Braganza et al. (2007) also did not require a communication link between the tugboats. Another adaptive control strategy was presented in Bui et al. (2010) that took the uncertainty of system parameters into account. In Esposito et al. (2008), an optimization based force/ torque allocation was employed and compared against a commutation based force/torque allocation strategy. In Bui and Kim (2011), position tracking control of ship berthing with assistance of autonomous tugboats was provided by using sliding mode control approach, while, a robust approach was presented in Ji et al. (2013). Recently in Tran and Im (2012), artificial neural networks were utilized to address the same problem. While several control aspects of the problem was researched, most of the above mentioned works required poses of the tugboats relative to the center 
of mass of the unactuated surface vessel to remain unchanged (i.e., static positioning was considered) which is usually not the case due to several disturbances. An attempt to relax the static positioning of the tugboats was discussed in Topp and Feemster (2010) for a simple one degree of freedom scenario. Unfortunately the extension of the result to manipulation of an unactuated surface vessel with multiple tugboats problem was unclear.

In this paper, a robust controller is proposed for the position tracking control of a large surface vessel manipulated by 6 unidirectional tugboats where the surface vessel was considered to be under the influence of added mass effects. The control problem is further complicated by the lack of accurate positions and orientations of tugboats. To illustrate the problem in hand, first the dynamic model of a 3 degree of freedom unactuated surface vessel manipulated by 6 uni-directional tugboats is given. Upon specification of the initial configurations of the uni-directional tugboats, the control is considered to be performed by 3 bi-directional tugboats where a force decomposition and commutation strategy is employed. Next, the open-loop error system is obtained where an uncertain input gain matrix, which includes uncertain inertia matrix of the surface vessel and uncertain thrust configuration matrix including uncertain possibly time-varying positions and orientations of tugboats, is obtained. A matrix decomposition is applied to initiate the control design. A robust controller, which does not require the configurations of the 3 bi-directional tugboats, is then proposed. Detailed stability analysis is presented where asymptotic tracking is ensured. Numerical simulations are performed where the positions and orientations of the tugboats are perturbed with sinusoidal terms are also presented to illustrate the performance of the proposed method.

\section{System model}

The dynamic model of a 3 degree of freedom unactuated surface vessel manipulated by six tugboats can be written as (Fossen, 1994, 2002; Skjetne et al., 2004; Arrichiello et al., 2006; Ihle et al., 2006; Fossen, 2011)

$M_{s} \dot{\nu}+D_{s}=F$
$\dot{x}=R \nu$

where $F=\left[F_{x}, F_{y}, M_{z}\right]^{\mathrm{T}} \in \mathbb{R}$ represents the total forces and moments applied to the vessel and acting on the center of mass of the vessel by the tugboats. The inertia matrix is denoted by $M_{s}(\nu)$ $\in \mathbb{R}^{3 \times 3}$ and obtained as (Fossen, 1994)

$M_{S}=M_{R B}+M_{A}$

where $M_{R B}(\nu) \in \mathbb{R}^{3 \times 3}$ denotes the positive definite, symmetric rigid body part of the inertia matrix while the effects due to added mass is represented by $M_{A}(\nu) \in \mathbb{R}^{3 \times 3}$. It should be noted that $M_{A}$ is not necessarily symmetric, resulting in a possibly non-symmetric $M_{s}$ (Fossen, 1994). However, in this study it is assumed that the added mass term does not lead to a rank deficiency in $M_{s}$ (i.e., $M_{s}$ is full rank). The vector $D_{s}(\nu) \in \mathbb{R}^{3}$ contains drag, damping, and other parasitic effects while $x(t)=\left[x_{p}(t), y_{p}(t), \psi(t)\right]^{\mathrm{T}} \in \mathbb{R}^{3}$ represents the composite inertial position $x_{p}, y_{p}$, and heading $\psi$ of the vessel while body fixed linear and angular velocity signals are represented by $\nu(t)=[u(t), v(t), \dot{\psi}(t)]^{\mathrm{T}} \in \mathbb{R}^{3}$. The rotation matrix is denoted by $R(\psi) \in S O(3)$ and has the following form:

$R(\psi)=\left[\begin{array}{ccc}\cos (\psi) & -\sin (\psi) & 0 \\ \sin (\psi) & \cos (\psi) & 0 \\ 0 & 0 & 1\end{array}\right]$

\subsection{Force decomposition and commutation strategy}

The unactuated vessel is moved via thrust inputs, provided from six tugboats in contact with the vessel's hull as illustrated in Fig. 1. Accordingly, $F$ in (1) is a result of the combined efforts provided from six tugboats and is expressed as

$F=B_{1} U_{1}$

where $U_{1}(t)=\left[u_{1 a}, u_{1 b}, u_{2 a}, u_{2 b}, u_{3 a}, u_{3 b}\right]^{\mathrm{T}} \in \mathbb{R}^{6}$ denotes a thrust input vector from six tugboats while the thrust configuration is

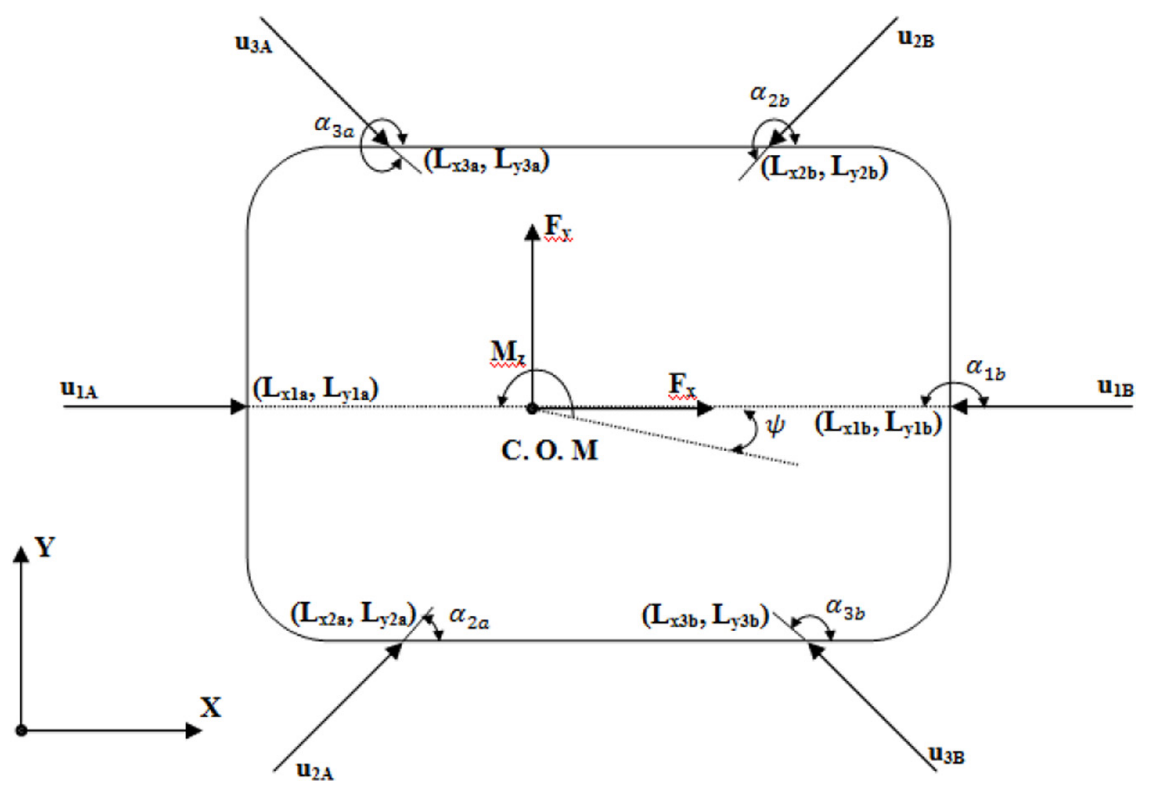

Fig. 1. System description showing the vessel frames. 
shown by $B_{1} \in \mathbb{R}^{3 \times 6}$ and has the following structure:

$$
B_{1}=\left[\begin{array}{ccc}
1 & 0 & 0 \\
-1 & 0 & 0 \\
C_{\alpha 2 a} & S_{\alpha 2 a} & L_{y 2 a} C_{\alpha 2 a}-L_{x 2 a} S_{\alpha 2 a} \\
C_{\alpha 2 b} & S_{\alpha 2 b} & L_{y 2 b} C_{\alpha 2 b}-L_{x 2 b} S_{\alpha 2 b} \\
C_{\alpha 3 a} & S_{\alpha 3 a} & -L_{y 3 a} C_{\alpha 3 a}+L_{x 3 a} S_{\alpha 3 a} \\
C_{\alpha 3 b} & S_{\alpha 3 b} & -L_{y 3 b} C_{\alpha 3 b}+L_{x 3 b} S_{\alpha 3 b}
\end{array}\right]^{\mathrm{T}}
$$

where $C_{\theta}=\cos (\theta)$ and $S_{\theta}=\sin (\theta)$. The opposite tugboats can then be placed as

$\alpha_{2 b}=\alpha_{2 a}+\pi \quad L_{x 2 b}=L_{x 2 a} \quad L_{y 2 b}=L_{y 2 a}$

$\alpha_{3 b}=\alpha_{3 a}-\pi \quad L_{x 3 b}=L_{x 3 a} \quad L_{y 3 b}=L_{y 3 a}$

which allows the force equation in (5) to be rewritten as

$F=B U$

where $U(t)=\left[u_{1}, u_{2}, u_{3}\right]^{\mathrm{T}} \in \mathbb{R}^{3}$ is the combined bi-directional control efforts from the opposing uni-directional tugboats with $u_{i}=u_{i a}-u_{i b}, \forall i=1,2,3$ and $B \in \mathbb{R}^{3 \times 3}$ is the thrust configuration matrix obtained as

$B=\left[\begin{array}{ccc}1 & C_{\alpha 2 a} & C_{\alpha 3 a} \\ 0 & S_{\alpha 2 a} & S_{\alpha 3 a} \\ 0 & L_{y 2 a} C_{\alpha 2 a}-L_{x 2 a} S_{\alpha 2 a} & -L_{y 3 a} C_{\alpha 3 a}+L_{x 3 a} S_{\alpha 3 a}\end{array}\right]$.

It is remarked that, similar to Braganza et al. (2007), the tugboats are considered to be placed according to the configurations in (7). In this study, unlike Braganza et al. (2007), we consider the configurations in (7) are the initial configurations and that they may vary after the motion starts. This relaxes the static positioning assumption in Braganza et al. (2007) and is an important novel departure from the existing literature.

In the subsequent sections, the control input $U(t)$ will be designed to obtain satisfactory tracking performance. The following commutation strategy can then be applied to $U(t)$ to specify uni-directional thrust effects provided by the bi-directional tugboats (de Queiroz and Dawson, 1996)

$u_{i a}=\frac{1}{2}\left(u_{i}+\sqrt{u_{i}^{2}+\epsilon_{0}^{2}}\right)$

$u_{i b}=\frac{1}{2}\left(-u_{i}+\sqrt{u_{i}^{2}+\epsilon_{0}^{2}}\right)$

for $i=1,2,3$ and where $\epsilon_{0} \in \mathbb{R}^{+}$denotes a controller parameter selected to obtain non-zero $u_{i a}(t)$ and $u_{i b}(t)$ to prevent the tugboats from losing contact with the vessel.

\subsection{Open-loop operation}

The time derivative of (2) is taken to obtain

$\ddot{x}=\dot{R} \nu+R \dot{\nu}$

which contains the time derivative of $R(\psi)$ that can be obtained as follows as a result of the special orthogonal structure of the rotation matrix:

$\dot{R}=R S_{3}$

where $S_{3}(\dot{\psi}) \in \mathbb{R}^{3 \times 3}$ is a skew-symmetric matrix defined as

$S_{3} \triangleq \dot{\psi}\left[\begin{array}{lll}0 & -1 & 0 \\ 1 & 0 & 0 \\ 0 & 0 & 0\end{array}\right]$.

After substituting (13) into (12), the right-hand side of (12) can be re-arranged as

$\ddot{x}=-R\left(M_{s}^{-1} D_{s}-S_{3} \nu\right)+R M_{s}^{-1} B U$ where (1) and (8) were utilized. A more compact form of the above model is obtained as follows by rewriting its right-hand side as

$\ddot{x}=h+G U$

with the functions $h(x, \nu) \in \mathbb{R}^{3}$ and $G(x, \nu) \in \mathbb{R}^{3 \times 3}$ defined in the following form:

$h \triangleq-R\left(M_{s}^{-1} D_{s}-S_{3} \nu\right)$

$G \triangleq R M_{s}^{-1} B$.

We would like to note that since $G(x, \nu)$ is a real matrix with nonzero leading principal minors, the following matrix decomposition is possible (Costa et al., 2003; Morse, 1993)

$G=S D U_{u}$

where $S(x, \nu) \in \mathbb{R}^{3 \times 3}$ represents a symmetric positive definite matrix, while a diagonal matrix with entries being \pm 1 and a unity upper triangular matrix are denoted by $D \in \mathbb{R}^{3 \times 3}$ and $U_{u}(x, \nu) \in \mathbb{R}^{3 \times 3}$, respectively. As a result of applying the above matrix decomposition to the models that are available in the literature, $D$ came out to be an identity matrix. Despite this, the derivations given in this paper will be presented for the general case where it is assumed that $D$ is available for control design (see Chen et al., 2008) for the precedence of this type assumption).

After taking the time derivative of (16), we obtain

$\dddot{x}=\varphi+S D U_{u} \dot{U}$

where (16) and (18) were utilized, and $\varphi(x, \dot{x}, \ddot{x}) \in \mathbb{R}^{3}$ is an auxiliary term defined as

$\varphi \triangleq \dot{h}+\dot{G} G^{-1}(\ddot{x}-h)$.

At this point, we would like to define the inverse of $S$ as $M(x, \nu) \in \mathbb{R}^{3 \times 3}$. It is remarked that $M$ is symmetric and positive definite because of symmetry and positive definiteness of $S$. In addition to this, the following bounds are valid for $M(x, \nu)$ :

$\underline{m}\|\chi\|^{2} \leq \chi^{T} M(\chi, \nu) \chi \leq \bar{m}\|\chi\|^{2} \quad \forall \chi \in \mathbb{R}^{3}$

where $m \in \mathbb{R}$ and $\bar{m}(x, \nu) \in \mathbb{R}$ denote a positive bounding constant and a positive non-decreasing bounding function, respectively.

The following equation can be obtained by multiplying both sides of (19) with $M$

$M \dddot{x}=f+D U_{u} \dot{U}$

where $f(x, \dot{x}, \ddot{x}) \triangleq M \varphi \in \mathbb{R}^{3}$.

\section{Error system development}

Ensuring a good tracking performance for inertial positions and heading of the vessel for a given reference trajectory and guaranteeing the boundedness of all signals under the closed-loop operation constitute our main control objectives. The subsequent control design is based on the availability of $x(t)$ and $\dot{x}(t)$ (i.e., fullstate feedback).

The output tracking error, $e_{1}(t) \in \mathbb{R}^{3}$, is defined as follows to quantify the tracking control objective:

$e_{1} \triangleq x_{d}-x$

where $x_{d}(t) \in \mathbb{R}^{3}$ is a smooth reference trajectory that is chosen in the sense that

$x_{d}(t) \in \mathcal{C}^{3}$ and $x_{d}^{(i)}(t) \in \mathcal{L}_{\infty}, \quad i=0,1,2,3$.

In order to eliminate the higher order time derivatives from the subsequent Lyapunov-based stability analysis, the auxiliary error signals, $e_{2}(t) \in \mathbb{R}^{3}$ and $r(t) \in \mathbb{R}^{3}$ are defined as follows:

$e_{2} \triangleq \dot{e}_{1}+e_{1}$ 
$r \triangleq \dot{e}_{2}+\Gamma e_{2}$

where $\Gamma \in \mathbb{R}^{3 \times 3}$ denotes a positive-definite, diagonal, constant gain matrix. The following expression is obtained by taking the time derivative of (26) and multiplying the result from left with $M$ :

$M \dot{r}=M\left(\dddot{x}_{d}+\ddot{e}_{1}+\Gamma \dot{e}_{2}\right)-f-D U_{u} \dot{U}$

where (22) and the time derivatives of (23) and (25) were utilized. The right-hand side of (27) can be re-arranged as

$M \dot{r}=-\frac{1}{2} \dot{M} r-e_{2}-D U_{u} \dot{U}+N$

where $N\left(x, \dot{x}, \ddot{x}, x_{d}, \dot{x}_{d}, \ddot{x}_{d}, \dddot{x}_{d}, t\right) \in \mathbb{R}^{3}$ is an auxiliary term defined as $N \triangleq M\left(\dddot{x}_{d}+\ddot{e}_{1}+\Gamma \dot{e}_{2}\right)-f+e_{2}+\frac{1}{2} \dot{M} r$.

The auxiliary function $N$ can be partitioned as a sum of two newly defined auxiliary terms denoted by $\bar{N}(t), \tilde{N}(t) \in \mathbb{R}^{3}$. These auxiliary terms are defined as

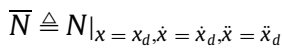

$\tilde{N} \triangleq N-\bar{N}$

After substituting the above definitions, the final form of openloop error system can be obtained as follows:

$M \dot{r}=-\frac{1}{2} \dot{M} r-e_{2}-D U_{u} \dot{U}+\tilde{N}+\bar{N}$

\section{Controller formulation}

Motivated by the subsequent stability analysis and based on the open-loop error system in (32), the control input $U(t)$ is designed as

$U=D K\left[e_{2}(t)-e_{2}\left(t_{0}\right)+\Gamma \int_{t_{0}}^{t} e_{2}(\sigma) d \sigma\right]+D \Pi$

where the auxiliary signal $\Pi(t) \in \mathbb{R}^{3}$ is generated according to the update law

$\dot{\Pi}(t)=C \operatorname{Sgn}\left(e_{2}(t)\right)$ with $\Pi\left(t_{0}\right)=0_{3}$.

In (33) and (34), $K, C \in \mathbb{R}^{3 \times 3}$ denote positive definite, diagonal, constant gain matrices while a vector of zeros is represented by $0_{3} \in \mathbb{R}^{3}$ and Sgn $(\cdot) \in \mathbb{R}^{3}$ is the vector signum function. The control gain is chosen as $K=I_{3}+k_{p} I_{3}+\operatorname{diag}\left\{k_{d, 1}, k_{d, 2}, 0\right\}$ where $k_{p}, k_{d, 1}, k_{d, 2}$ $\in \mathbb{R}$ are positive, constant controller gains, the notation $\operatorname{diag}\{\cdot\}$ represents a diagonal matrix, and $I_{3} \in \mathbb{R}^{3 \times 3}$ is the standard identity matrix. The following closed-loop error system is obtained by substituting the time derivative of (33) into (32) and then adding and subtracting $D K r(t)$

$$
\begin{aligned}
M \dot{r}= & -\frac{1}{2} \dot{M} r-e_{2}-K r+\tilde{N}+\bar{N} \\
& -D\left(U_{u}-I_{3}\right) D K r-D U_{u} D C \operatorname{Sgn}\left(e_{2}\right)
\end{aligned}
$$

where (34) and the fact that $D D=I_{3}$ were utilized.

Before presenting the accompanying stability analysis, a more detailed examination of the last two terms of (35) are given. The $D\left(U_{u}-I_{3}\right) D K r$ term can be rewritten as

$D\left(U_{u}-I_{3}\right) D K r=\left[\begin{array}{c}\Lambda_{1} \\ \Lambda_{2} \\ 0\end{array}\right]+\left[\begin{array}{c}\Phi_{1} \\ \Phi_{2} \\ 0\end{array}\right]$

where the auxiliary signals $\Lambda_{1}(t), \Lambda_{2}(t), \Phi_{1}(t), \Phi_{2}(t) \in \mathbb{R}$ are defined as

$\Lambda_{1} \triangleq d_{1} d_{2} k_{2} \tilde{U}_{u_{1,2}} r_{2}+d_{1} d_{3} k_{3} \tilde{U}_{u_{1,3}} r_{3}$

$\Lambda_{2} \triangleq d_{2} d_{3} k_{3} \tilde{U}_{u_{2,3}} r_{3}$

$\Phi_{1} \triangleq d_{1} d_{2} k_{2} \bar{U}_{u_{1,2}} r_{2}+d_{1} d_{3} k_{3} \bar{U}_{u_{1,3}} r_{3}$
$\Phi_{2} \triangleq d_{2} d_{3} k_{3} \bar{U}_{u_{23}} r_{3}$

with the following definitions of $\bar{U}_{u_{1,2}}(t), \bar{U}_{u_{1,3}}(t), \bar{U}_{u_{2,3}}(t), \tilde{U}_{u_{1,2}}(t)$, $\tilde{U}_{u_{1,3}}(t), \tilde{U}_{u_{2,3}}(t) \in \mathbb{R}$ as

$\left.\bar{U}_{u_{i, j}} \triangleq U_{u_{i, j}}\right|_{x=x_{d}, \dot{x}=\dot{x}_{d}}$

$\tilde{U}_{u_{i, j}} \triangleq U_{u_{i, j}}-\bar{U}_{u_{i, j}}$

where $U_{u_{i, j}}(x, \nu) \in \mathbb{R}$ represents the $(i, j)$-th entry of $U_{u}(x, \nu)$. From (29), it can be seen that $\Lambda_{2}(t)$ depends on $k_{3}$, and from (37), it is clear that, $\Lambda_{1}(t)$ depends on $k_{3}$ and $k_{2}$. From (39) and (40), it can also be seen that $\Phi_{1}(t)$ depends on $k_{3}$ and $k_{2}$ while $\Phi_{2}(t)$ depends on $k_{3}$.

On the other hand, the following decomposition can be applied to $D U_{u} D C \operatorname{Sgn}\left(e_{2}\right)$ term:

$D U_{u} D C \operatorname{Sgn}\left(e_{2}\right)=\left[\Psi^{T}, 0\right]^{T}+\Theta$

where two newly defined auxiliary terms denoted by $\Psi(t) \in \mathbb{R}^{2}$ and $\Theta(t) \in \mathbb{R}^{3}$ have the following forms:

$\left[\Psi^{T}, 0\right]^{T}=D\left(U_{u}-\bar{U}_{u}\right) D C \operatorname{Sgn}\left(e_{2}\right)$

$\Theta \triangleq D \bar{U}_{u} D C \operatorname{Sgn}\left(e_{2}\right)$

where $\left.\bar{U}_{u}\left(x_{d}, \dot{x}_{d}\right) \triangleq U_{u}\right|_{x=x_{d}} \dot{x}=\dot{x}_{d} \in \mathbb{R}^{3 \times 3}$ is a function of reference trajectory and its time derivative, and $\Psi_{i}(t) \in \mathbb{R}, \quad i=1,2$ and $\Theta_{i}(t) \in \mathbb{R}, i=1,2,3$, are defined as

$\Psi_{i}=d_{i} \sum_{j=i+1}^{3} d_{j} C_{j} \tilde{U}_{u_{i, j}} \operatorname{sgn}\left(e_{2, j}\right)$

$\Theta_{i}=d_{i} \sum_{j=i}^{3} d_{j} C_{j} \bar{U}_{u_{i j}} \operatorname{sgn}\left(e_{2, j}\right)$

Remark 1. The following upper bounds can be developed by utilizing the Mean Value Theorem (Khalil, 2002)

$\|\tilde{N}(\cdot)\| \leq \rho_{\tilde{N}}(\|z\|)\|z\|$

$\left\|\tilde{U}_{i, j}(\cdot)\right\| \leq \rho_{i, j}(\|z\|)\|z\|$

where $\rho_{\tilde{N}}(\cdot), \rho_{i, j}(\cdot) \in \mathbb{R}$ are non-negative, globally invertible, nondecreasing functions of their arguments, and $z(t) \in \mathbb{R}^{9}$ is defined as

$z \triangleq\left[\begin{array}{lll}e_{1}^{T} & e_{2}^{T} & r^{T}\end{array}\right]^{T}$.

It can be seen from (30) and (41) that $\bar{N}(t)$ and $\bar{U}_{u_{i j}}(t)$ can be upper bounded as

$\left|\bar{N}_{i}(t)\right| \leq \zeta_{\bar{N}_{i}}$

$\left|\bar{U}_{u_{i, j}}(t)\right| \leq \zeta_{\bar{U}_{u_{i, j}}}$

where $\zeta_{\bar{N}_{i}}, \zeta_{\bar{U}_{u_{i j}}} \in \mathbb{R}$ are positive bounding constants. Based on (37)-(40), (46) and (47), following upper bounds can be obtained:

$\left|\Lambda_{i}\right| \leq \rho_{\Lambda_{i}}(\|z\|)\|z\|$

$\left|\Phi_{i}\right| \leq \zeta_{\Phi_{i}}\|z\|$

$\left|\Psi_{i}\right| \leq \rho_{\Psi_{i}}(\|z\|)\|z\|$

for $i=1,2$ and the following upper-bound can also be obtained

$\left|\Theta_{i}\right| \leq \zeta_{\Theta_{i}}$

for $i=1,2,3$ where (48)-(52) were utilized. From (56), it is clear that $\|\Theta\| \leq \zeta_{\Theta}$ is provided for some positive bounding constant $\zeta_{\Theta} \in \mathbb{R}$, and from (53)-(55), we obtain

$\left|\Lambda_{i}\right|+\left|\Phi_{i}\right|+\left|\Psi_{i}\right| \leq \rho_{i}(\|z\|)\|z\|$ 
where $\rho_{i}(\|z\|) \in \mathbb{R} \quad i=1,2$, are non-negative, globally invertible, non-decreasing functions satisfying

$\rho_{\Lambda_{i}}+\rho_{\Psi_{i}}+\zeta_{\Phi_{i}} \leq \rho_{i}$.

After this point, we can continue with the Lyapunov-based boundedness and convergence analysis of the proposed robust controller design.

\section{Stability analysis}

Proving the boundedness of the error signals under the closedloop operation using Lyapunov-type stability analysis is the first purpose of this section. Then, we will present a lemma and obtain an upper bound for the integral of the absolute values of the entries of the time derivative of $e_{2}(t)$ by making use of the boundedness result. This upper bound will be utilized in another lemma to prove the non-negativity of a Lyapunov-like function. Finally, asymptotic stability of the overall closed-loop system will be proven by using the results of this lemma.

Theorem 1 (Boundedness proof). The controller in (33) and(34) guarantee the boundedness of the closed-loop system including the error signals in (23), (25) and(26) provided that the controller gains $k_{d, 1}, k_{d, 2}$ and $k_{p}$ are chosen large enough compared to the initial conditions of the system and the following condition is satisfied:

$\lambda_{\min }(\Gamma) \geq \frac{1}{2}$

where $\lambda_{\min }(\Gamma)$ is the minimum eigenvalue of the gain matrix $\Gamma$.

Proof. The non-negative function $V_{1}(z) \in \mathbb{R}$ is defined as ${ }^{1}$

$V_{1} \triangleq \frac{1}{2} e_{1}^{T} e_{1}+\frac{1}{2} e_{2}^{T} e_{2}+\frac{1}{2} r^{T} M r$

The Lyapunov function in (60) can be lower and upper bounded as follows by utilizing (21):

$\lambda_{1}\|z\|^{2} \leq V_{1}(z) \leq \lambda_{2}\|z\|^{2}$

with $\lambda_{1} \triangleq \frac{1}{2} \min \{1, m\}$ and $\lambda_{2} \triangleq \max \left\{1, \frac{1}{2} \bar{m}(\|z\|)\right\}$ and the terms $m, \bar{m}(\|z\|)$ were defined in (21) and $z(t)$ was defined in (50). The following inequality is obtained by taking the time derivative of (60), making necessary substitutions, and then performing straightforward mathematical manipulations and grouping

$\dot{V}_{1} \leq-\beta_{1} V_{1}+\delta_{1}$

where $\beta_{1}, \delta_{1} \in \mathbb{R}$ denote positive constants. The boundedness of $V_{1}(t)$ can be obtained from (60) and (62) (i.e., $V_{1}(t) \in \mathcal{L}_{\infty}$ ), therefore $e_{1}(t)$, $e_{2}(t)$ and $r(t)$ are uniformly ultimately bounded. Utilization of the standard signal chasing arguments allows us to prove the boundedness of all remaining signals under the closed-loop operation. $\square$

Lemma 1. Provided that $e_{2}(t)$ and $\dot{e}_{2}(t)$ are bounded, the following expression for the upper bound of the integral of the absolute value of the $i$-th entry of $\dot{e}_{2}(t) i=1,2,3$ can be obtained

$\int_{t_{0}}^{t}\left|\dot{e}_{2, i}(\sigma)\right| d \sigma \leq \kappa_{1}+\kappa_{2} \int_{t_{0}}^{t}\left|e_{2, i}(\sigma)\right| d \sigma+\left|e_{2, i}\right|$

where $\kappa_{1}, \kappa_{2} \in \mathbb{R}$ are some positive bounding constants.

Proof. Readers can refer to Bidikli et al. (2013a) for the proof. $\square$

Remark 2. As a result of the fact that $\bar{U}_{u}(t)$ being unity upper triangular, $\Theta(t)$ in (45) can be rewritten as

$\Theta=\left(I_{3}+\Omega\right) C \operatorname{Sgn}\left(e_{2}\right)$

${ }^{1}$ A highlight of the proof is given. The detailed proof for the $n$-th order version of the system, which can straightforwardly be adopted this proof, can be found in Bidikli et al. (2013a). where $\Omega(t) \triangleq D\left(\bar{U}_{u}-I_{3}\right) D \in \mathbb{R}^{3 \times 3}$ is a strictly upper triangular matrix. Since it is a function of the reference trajectory and its time derivatives, its entries, denoted by $\Omega_{i, j}(t) \in \mathbb{R}$, are bounded in the sense that

$\left|\Omega_{i, j}\right| \leq \zeta_{\Omega_{i, j}}$

where $\zeta_{\Omega_{i, j}} \in \mathbb{R}$ are positive bounding constants.

Lemma 2. Consider the term

$L \triangleq r^{T}\left[\bar{N}-\left(I_{3}+\Omega\right) C \operatorname{Sgn}\left(e_{2}\right)\right]$.

Provided that the entries of the control gain matrix $C$ are chosen to satisfy in an orderly fashion

$C_{3} \geq \zeta_{\bar{N}_{3}}\left(1+\frac{\kappa_{2}}{\Gamma_{3}}\right)$

$C_{2} \geq\left(\zeta_{\bar{N}_{2}}+\zeta_{\Omega_{2,3}} C_{3}\right)\left(1+\frac{\kappa_{2}}{\Gamma_{2}}\right)$

$C_{1} \geq\left(\zeta_{\bar{N}_{1}}+\zeta_{\Omega_{1,2}} C_{2}+\zeta_{\Omega_{1,3}} C_{3}\right)\left(1+\frac{\kappa_{2}}{\Gamma_{1}}\right)$

where $\Gamma_{i}$ for $i=1,2,3$ denotes the $i$-th diagonal element of $\Gamma$, then it can be concluded that

$\int_{t_{0}}^{t} L(\sigma) d \sigma \leq \zeta_{L}$

where $\zeta_{L} \in \mathbb{R}$ is a positive bounding constant defined as

$\zeta_{L} \triangleq \kappa_{1} \sum_{i=1}^{2} \sum_{j=i+1}^{3} \zeta_{\Omega_{i, j}} C_{j}+\kappa_{1} \sum_{i=1}^{3} \zeta_{\bar{N}_{i}}+\sum_{i=1}^{3} C_{i}\left|e_{2, i}\left(t_{0}\right)\right|$.

Proof. Readers can refer to Bidikli et al. (2013a) for the proof. $\square$

Theorem 2 (Asymptotic convergence proof). Given the dynamic model in (1) and (2), the controller of (33) and (34) ensures the convergence of the tracking error $e_{1}(t)$ asymptotically to the origin in the sense that

$\left\|e_{1}(t)\right\| \rightarrow 0$ as $t \rightarrow+\infty$

provided that $\Gamma$ is chosen to satisfy (59), the entries of $C$ are chosen to satisfy (67)-(69), and $k_{p}, k_{d, 1}, k_{d, 2}$ are chosen large enough.

Proof. See Appendix for the proof. $\square$

Remark 3. The entries of the control gain matrix $C$ are required to satisfy (67) which depends on the constant upper bounds of uncertain system functions, and the entries of the control gain matrix $K$ are required to be chosen large enough compared to the initial conditions of the system. While this may seem like a weakness of the proposed controller, we will address this issue by utilizing the self-tuning strategy that we recently designed in Bidikli et al. (2013b, 2014) for the family of the controllers in Xian et al. (2004) as an add-on to adjust the entries of $C$ and $K$. Specifically, the entries of gain matrices $C$ and $K$ are updated according to

$C_{i}(t)=\left|e_{2 i}(t)\right|-\left|e_{2 i}(t)\right|+\Gamma_{i} \int_{0}^{t}\left|e_{2 i}(\sigma)\right| d \sigma$

$K_{i}(t)=k_{c i}+\frac{1}{2} e_{2 i}^{2}(t)+\Gamma_{i} \int_{0}^{t} e_{2 i}^{2}(\sigma) d \sigma$ 

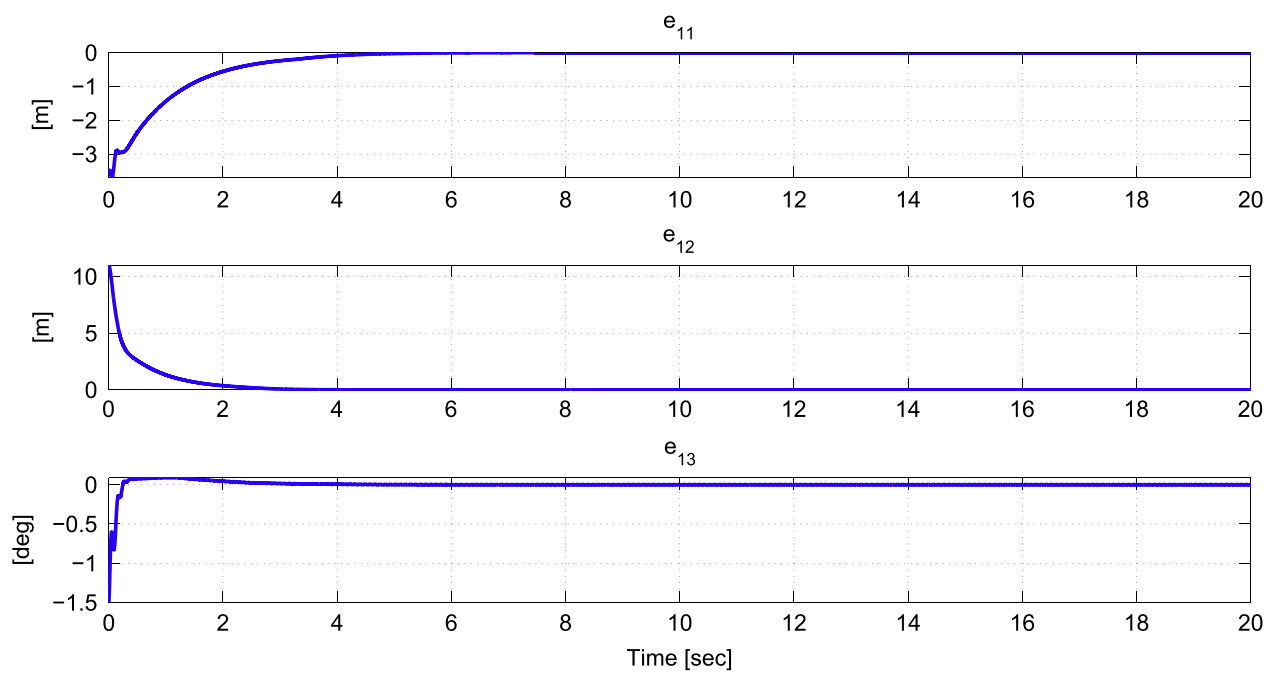

Fig. 2. Tracking errors.

for $i=1,2,3$ where $k_{c i} \in \mathbb{R}$ are the positive constant parts of the time-varying gain.

\section{Simulation results}

The performance of the proposed robust controller was demonstrated via numerical simulations. The ship model in (1) was utilized with the following inertia matrix (Fossen, 2002)

$M_{s}(x)=\left[\begin{array}{ccc}m+X_{\dot{u}} & 0 & 0 \\ 0 & n_{a} & n_{d} \\ 0 & n_{c} & n_{b}\end{array}\right]$

where the auxiliary terms $n_{a}, n_{b}, n_{c}$ and $n_{d}$ are defined as

$n_{a}=m-Y_{\dot{v}}, \quad n_{b}=I_{z}-N_{\dot{r}}$,

$n_{c}=m x_{g}-N_{\dot{v}}, \quad n_{d}=m x_{g}-Y_{\dot{r}}$.

In the above inertia matrix, constant terms $Y_{\dot{r}}$ and $N_{\dot{v}}$ are selected as

$Y_{\dot{r}}=0.0, \quad N_{\dot{v}}=-1.0$

to reflect the effects of added mass which result in a nonsymmetric $^{2}$ inertia matrix. The damping matrix has the following form

$D_{s}(\nu)=\left[\begin{array}{ccc}d_{11} & 0 & 0 \\ 0 & d_{22} & d_{23} \\ 0 & d_{32} & d_{33}\end{array}\right]$

with its entries defined as

$d_{11}=-X_{u}+\left(-X_{|u| u}\left|u_{r}\right|-X_{\text {иии }} u_{r}^{2}\right)$

$d_{22}=-Y_{v}+\left(-Y_{|v| v}\left|u_{r}\right|-Y_{r v}|\dot{\psi}|\right)$

$d_{33}=-N_{r}+\left(-Y_{|v| v}\left|u_{r}\right|-Y_{|r| v \mid}|\dot{\psi}|\right)$

$d_{23}=-Y_{r}+\left(-Y_{|v| r}\left|u_{r}\right|-Y_{|r| v}|\dot{\psi}|\right)$

$d_{32}=-N_{v}+\left(-N_{|v| v}\left|v_{r}\right|-N_{r v}|\dot{\psi}|\right)$.

\footnotetext{
${ }^{2}$ In the other control approaches about this subject available in the literature, especially in Braganza et al. (2007), the inertia matrix was selected as a symmetric matrix (i.e., same values were selected for $Y_{\dot{r}}$ and $N_{\dot{v}}$ in (77)). In our study, the inertia matrix was selected to be non-symmetric. This is one of the important advantages of our controller.
}

The desired vessel position was given as

$x_{d}(t)=\left[\begin{array}{l}10 \sin (0.1 t)[\mathrm{m}] \\ 10 \cos (0.1 t)[\mathrm{m}] \\ -0.1 t[\mathrm{rad}]\end{array}\right]$.

The initial positions were set $x(0)=\left[0.3,2.5,-\frac{\pi}{4}\right]^{T}$ and the initial velocities were $v(0)=0_{3}$. The control gains $K$ and $C$ were obtained via the self-tuning strategy in (73) and (74) as

$K=\operatorname{diag}\{168.2,112.3,118.6\}, \quad C=\operatorname{diag}\{3.2,6.4,5.1\}$

and the other control gain $\Gamma$ and $\epsilon_{0}$ are selected as

$\Gamma=\operatorname{diag}\{1,5,2.3\}, \quad \epsilon_{0}=\sqrt{5}$.

In order to obtain a proper time-dependent nature for the tugboats' positions without losing their contact with the vessel's hull, the tugboats were positioned at the following locations with respect to the center of mass of the vessel

$L_{1 a}=[-0.5,0.1 \sin (t)]$

$L_{1 b}=[0.5,0.1 \sin (t)]$

$L_{2 a}=[-0.25+0.5 \sin (t),-0.145]$

$L_{2 b}=[-0.25+0.5 \sin (t), 0.145]$

$L_{3 a}=[0.1 \sin (t), 0.145]$

$L_{3 b}=[0.1 \sin (t),-0.145]$

while the incident angle of each tugboat with respect to the vessel's hull was selected as follows

$\alpha_{1 a}=(\pi / 180) \sin (t)$

$\alpha_{1 b}=\pi-(\pi / 180) \sin (t)$

$\alpha_{2 a}=\pi / 2+(\pi / 180) \sin (t)$

$\alpha_{2 b}=\alpha_{2 a}+(\pi / 180) \sin (t)$

$\alpha_{3 a}=3 \pi / 2+(\pi / 180) \sin (t)$

$\alpha_{3 b}=\alpha_{3 a}-\pi+(\pi / 180) \sin (t)$.

In (87) and (88), time-varying sinusoidal perturbations are added to demonstrate disturbance effects.

The position tracking errors and the control inputs are shown in Figs. 2-5, respectively. From Fig. 2, it can be clearly seen that unactuated surface vessel tracked the desired composite inertial positions and heading successfully which demonstrates that our tracking objective was successfully met. 


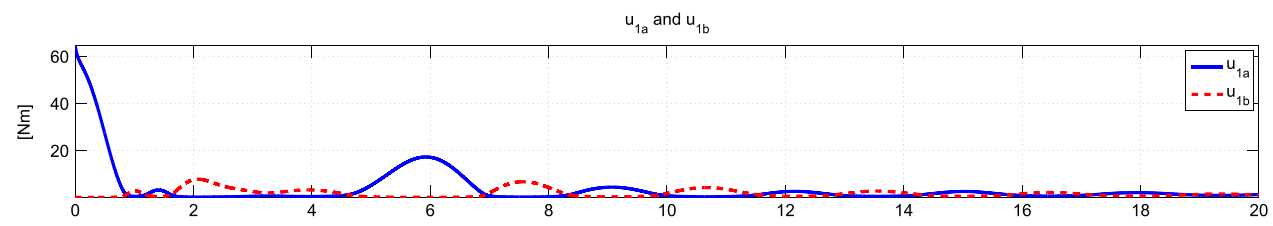

$\mathrm{u}_{2 \mathrm{a}}$ and $\mathrm{u}_{2 \mathrm{~b}}$

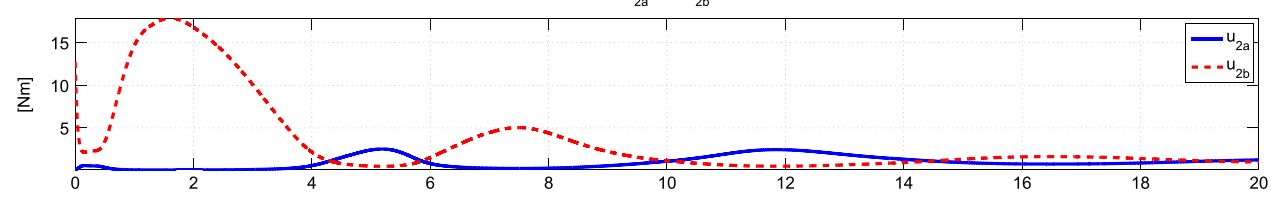

$u_{3 a}$ and $u_{3 b}$

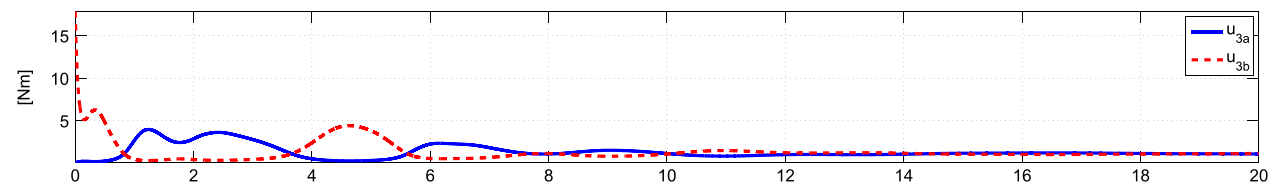

Fig. 3. Control torques.
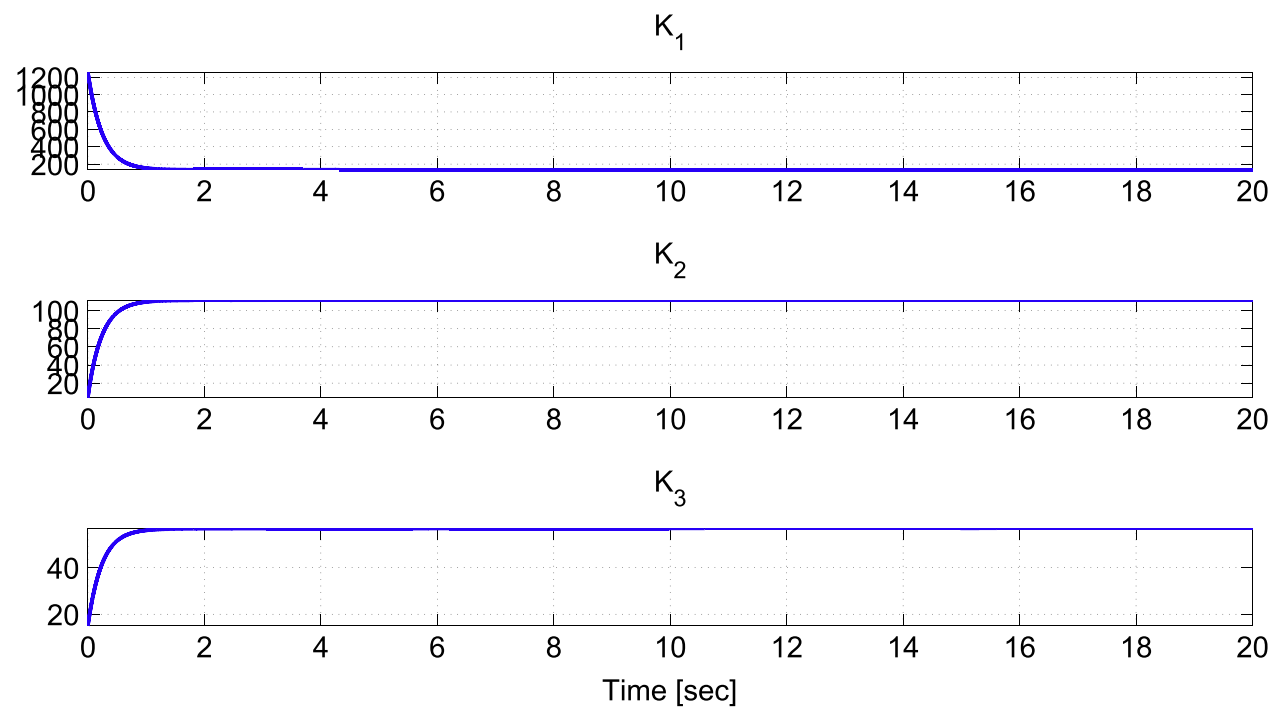

Fig. 4. Time varying gains $\mathrm{Ki}$ for $i=1 ; 2 ; 3$.
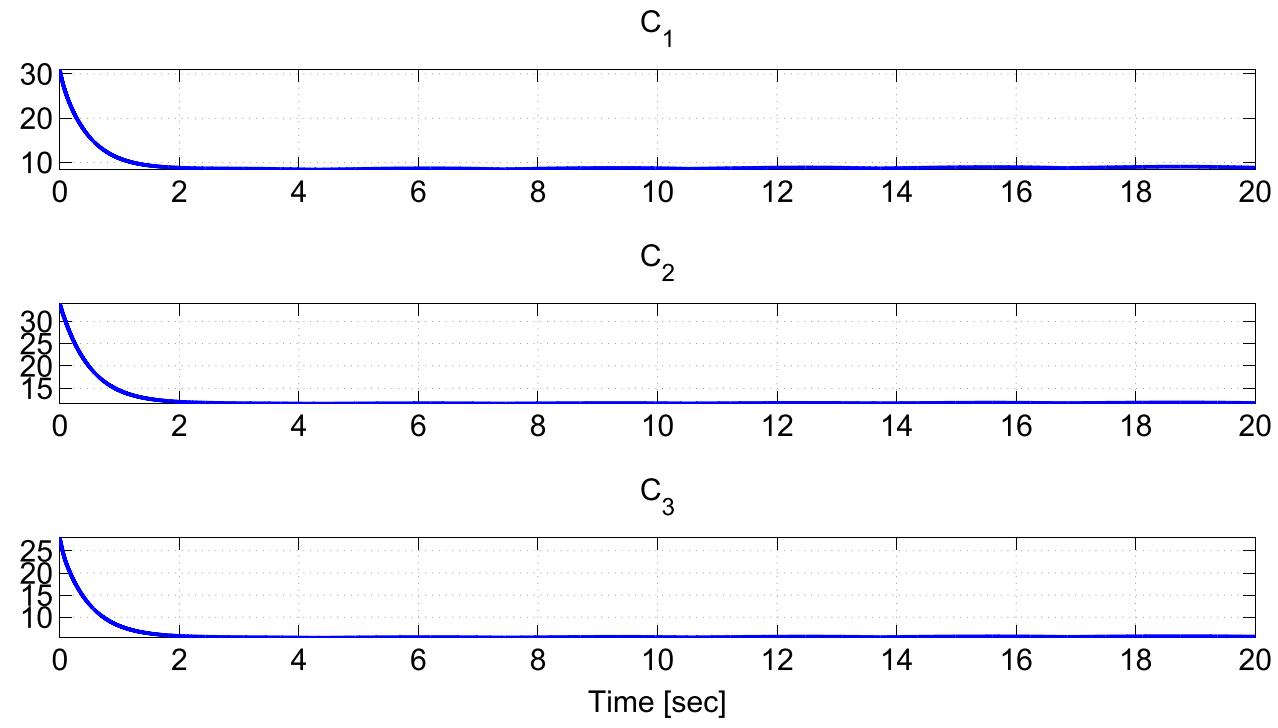

Fig. 5. Time varying gains $\mathrm{Ci}$ for $i=1 ; 2 ; 3$. 


\section{Conclusion}

In this paper, a robust controller for an unactuated surface vessel manipulated by autonomous tugboats was designed. The controller was designed under the restriction that the dynamic model of the surface vessel is uncertain. Furthermore, unlike the similar works in the literature, the surface vessel was considered to be under the influence of added mass effects which resulted in a non-symmetric inertia matrix. The control problem is further complicated by the lack of accurate positions and orientations of tugboats. As a result of these issues, the resulting open-loop error system had an uncertain input gain matrix. A matrix decomposition method was applied and a robust controller was designed. The stability of the closed-loop system was investigated via detailed Lyapunov-type tools where asymptotic tracking was proven. Numerical simulations were performed where the positions and the orientations of the tugboats were considered to be disturbed by sinusoidal perturbations. Satisfactory tracking performance was obtained.

The novelties of the proposed control design and the accompanying stability analysis can be listed as:

- Different from the existing works on this application nonsymmetric inertial added mass effects were considered and dealt with.

- While positions and orientations of the uni-directional tugboats were specified and combined models of the opposite tugboats were obtained, these positions and orientations were considered to be available initially and then they were allowed to vary. This was demonstrated in the numerical simulations and can be seen from (87) and (88). It is noted that Braganza et al. (2007) did not allow positions and orientations of the tugboats to vary.

- The self-tuning strategy (Bidikli et al., 2013b, 2014) was employed as an add-on in the numerical simulations to ease the control gain tuning process.

\section{Acknowledgments}

Works of E. Tatlicioglu and E. Zergeroglu are funded by The Scientific and Technological Research Council of Turkey via grant number 113E147.

\section{Appendix A. Asymptotic convergence proof}

The auxiliary function $P(t) \in \mathbb{R}$ is defined as

$P \triangleq \zeta_{L}-\int_{t_{0}}^{t} L(\sigma) d \sigma$.

where the terms $\zeta_{L}$ and $L(t)$ were defined in (66) and (71), respectively. When the entries of the control gain matrix $C$ are chosen to satisfy (67)-(69), from the proof of Lemma 2 in Bidikli et al. (2013a), we can conclude that $P(t)$ is non-negative.

At this stage, consider the Lyapunov function $V_{2}(z, t) \in \mathbb{R}$ defined as

$V_{2} \triangleq V_{1}+P$

where $s(t) \in \mathbb{R}^{10}$ is defined as

$s \triangleq\left[\begin{array}{ll}z^{T} & \sqrt{P}\end{array}\right]^{T}$

and $V_{1}(z) \in \mathbb{R}$ was defined in (60). The Lyapunov function in (91) can be lower and upper bounded as follows by utilizing (21)

$W_{1}(s) \leq V_{2}(s, t) \leq W_{2}(s)$ where $W_{1}(s), W_{2}(s) \in \mathbb{R}$ are defined as

$W_{1} \triangleq \lambda_{1}\|s\|^{2}, W_{2} \triangleq \lambda_{2}(\|z\|)\|s\|^{2}$.

Taking the time derivative of $V_{2}$, utilizing the time derivative of (70), canceling common terms yields

$$
\begin{aligned}
\dot{V}_{2}= & -e_{1}^{T} e_{1}+e_{1}^{T} e_{2}-e_{2}^{T} \Gamma e_{2}-r^{T} r \\
& +\left[r^{T} \tilde{N}-k_{p} r^{T} r\right] \\
& +\left[-\sum_{i=1}^{m-1} r_{i}\left(\Lambda_{i}+\Psi_{i}+\Phi_{i}\right)-\sum_{i=1}^{2} k_{d, i} r_{i}^{2}\right]
\end{aligned}
$$

which can be rearranged to have the following form:

$$
\begin{aligned}
\dot{V}_{2} \leq & -\frac{1}{2}\left\|e_{1}\right\|^{2}-\left(\lambda \min (\Gamma)-\frac{1}{2}\right)\left\|e_{2}\right\|^{2}-r^{T} r \\
& +\frac{\rho_{\tilde{N}}^{2}(\|z\|)}{4 k_{p}}\|z\|^{2}+\sum_{i=1}^{2} \frac{\rho_{i}^{2}(\|z\|)}{4 k_{d, i}}\|z\|^{2} \\
& \leq-\left(\lambda_{3}-\frac{\rho_{\tilde{N}}^{2}(\|z\|)}{4 k_{p}}-\sum_{i=1}^{2} \frac{\rho_{i}^{2}(\|z\|)}{4 k_{d, i}}\right)\|z\|^{2}
\end{aligned}
$$

where $\lambda_{3} \triangleq \min \left\{\frac{1}{2}, \lambda_{\min }(\Gamma)-\frac{1}{2}\right\}$. When the controller gains $k_{p}, k_{d, 1}$, $k_{d, 2}$ are selected large enough such that the regions defined by $\mathcal{D}_{z}$ $\triangleq\{z:\|z\| \leq \mathcal{R}\}$ and $\mathcal{D}_{s} \triangleq\{s:\|s\| \leq \mathcal{R}\}$ with $\mathcal{R}$ being defined as

$\mathcal{R}=\min \left\{\rho_{\tilde{N}}^{-1}\left(2 \sqrt{k_{p} \frac{1-\beta_{2}}{3}}\right), \quad \rho_{i}^{-1}\left(2 \sqrt{k_{d, i} \frac{1-\beta_{2}}{3}}\right)\right\}$

for $i=1,2$, are non-empty. From (95), (96) and the definition of $s$, one can then restate

$\dot{V}_{2} \leq-\beta_{2}\|z\|^{2} \triangleq W(s) \quad \forall s \in \mathcal{D}_{s}$

where $\beta_{2} \in \mathbb{R}$ is a positive constant that satisfies $0 \leq \beta_{2}<1$. From (90) and (97), it is obvious that $V_{2}(t) \in \mathcal{L}_{\infty}$, and from the proof of Theorem 1, we concluded that all signals in the closed-loop error system are bounded and furthermore, from the boundedness of $\dot{W}(s)$, we can state $W(s)$ is uniformly continuous.

Based on the definition of $\mathcal{D}_{s}$, another region, $\mathcal{S}$, can be defined in the following form:

$$
\begin{aligned}
\mathcal{S} & \triangleq\left\{s \in \mathcal{D}_{s}: W_{2}(s)<\lambda_{2}\left(\rho_{\tilde{N}}^{-1}\left(2 \sqrt{k_{p} \frac{1-\beta_{2}}{m}}\right)\right)^{2}\right\} \\
& \cap\left\{s \in \mathcal{D}_{s}: W_{2}(s)<\lambda_{2}\left(\rho_{1}^{-1}\left(2 \sqrt{k_{d, 1} \frac{1-\beta_{2}}{3}}\right)\right)^{2}\right\} \\
& \cap\left\{s \in \mathcal{D}_{s}: W_{2}(s)<\lambda_{2}\left(\rho_{2}^{-1}\left(2 \sqrt{k_{d, 2} \frac{1-\beta_{2}}{3}}\right)\right)^{2}\right\} .
\end{aligned}
$$

A direct application of Theorem 8.4 in Khalil (2002) can be used to prove that $\|z(t)\| \rightarrow 0$ as $t \rightarrow+\infty \forall s\left(t_{0}\right) \in \mathcal{S}$. Based on the definition of $z(t)$, it is easy to show that $\left\|e_{1}(t)\right\|,\left\|e_{2}(t)\right\|,\|r(t)\| \rightarrow 0$ as $t \rightarrow+\infty$ $\forall s\left(t_{0}\right) \in \mathcal{S}$. From (26), it is clear that $\left\|\dot{e}_{2}(t)\right\| \rightarrow 0$ as $t \rightarrow+\infty$ $\forall s\left(t_{0}\right) \in \mathcal{S}$. By utilizing (25), it can be proven that $\left\|\dot{e}_{1}(t)\right\| \rightarrow 0$ as $t \rightarrow+\infty, \forall s\left(t_{0}\right) \in \mathcal{S}$. Note that the region of attraction can be made arbitrarily large to include any initial conditions by choosing the controller gains $k_{p}, k_{d, 1}$ and $k_{d, 2}$. This fact implies that the stability result obtained by the proposed method is semi-global.

\section{References}

Arrichiello, F., Chiaverini, S., Fossen, T.I., 2006. Formation control of underactuated surface vessels using the null-space-based behavioral control, in: Proceedings of IEEE International Conference on Robotics and Automation, Orlando, FL, USA pp. 5942-5947.

Bidikli, B., Tatlicioglu, E., Zergeroglu, E., Bayrak, A., 2013. An Asymptotically Stable Continuous Robust Controller for a Class of Uncertain MIMO Nonlinear Systems, Tech. Rep. 1301.5483, ArXiv e-prints January. 
Bidikli, B., Tatlicioglu, E., Bayrak, A., Zergeroglu, E., 2013. A new robust 'integral of sign of error' feedback controller with adaptive compensation gain, in: Proceedings of IEEE International Conference on Decision and Control, Florence, Italy, pp. 3782-3787.

Bidikli, B., Tatlicioglu, E., Zergeroglu, E., 2014. A self tuning RISE controller formulation, in: Proceedings of American Control Conference, Portland, OR, USA pp. 5608-5613.

Braganza, D., Feemster, M., Dawson, D., 2007. Positioning of large surface vessels using multiple tugboats, in: Proceedings of American Control Conference, New York, NY, USA, pp. 912-917.

Bui, V., Kim, Y., 2011. Development of constrained control allocation for ship berthing by using autonomous tugboats. J. Control Autom. Syst. 9 (6) 1203-1208.

Bui, V., Kawai, H., Kim, Y., Lee, K., 2010. A ship berting system design with four tug boats. J. Mech. Sci. Technol. 25 (5), 1257-1264.

Chen, J., Behal, A. Dawson, D.M., 2008. Robust feedback control for a class of uncertain MIMO nonlinear systems. IEEE Trans. Autom. Control 53 (2), 591-596.

Costa, R.R., Hsu, L., Imai, A.K., Kokotovic, P., 2003. Lyapunov-based adaptive control of MIMO systems. Automatica 39 (7), 1251-1257.

de Queiroz, M.S., Dawson, D.M., 1996. Nonlinear control of active magnetic bearings: a backstepping approach. IEEE Trans. Control Syst. Technol. 4 (5), 545-552.

Esposito, J.M., Feemster, M., Smith, E., 2008. Cooperative manipulation on the water using a swarm autonomous tugboats, in: Proceedings of IEEE International Conference on Robotics and Automation, Pasadena, CA, USA, pp. 1501-1506.

Feemster, M.G., Esposito, J.M., 2011. Comprehensive framework for tracking contro and thrust allocation for a highly overactuated autonomous surface vessel. J. Field Robot. 28 (1), 80-100.

Feemster, M., Esposito, J.M., Nicholson, J., 2006. Manipulation of large objects by swarms of autonomous marine vehicles: Part i-rotation, in: Proceedings of Southeastern Symposium on System Theory, Cooksville, TN, USA, pp. 255-259.
Fossen, T.I., 1994. Guidance and Control of Ocean Vehicles. John Wiley and Sons, New York, NY, USA.

Fossen, T.I., 2002. Marine Control Systems: Guidance, Navigation, and Control of Ships, Rigs and Underwater Vehicles, Marine Cybernetics AS, Trondheim, Norway.

Fossen, T.I., 2011. Handbook of Marine Craft Hydrodynamics and Motion Control. John Wiley \& Sons, Hoboken, NJ, USA.

Ihle, I.-A.F., Jouffroy, J., Fossen, T.I., 2006. Formation control of marine surface craft: a Lagrangian approach. IEEE J. Ocean Eng. 31 (4), 922-934.

Ji, S.-W., Bui, V.P., Balachandran, B., Kim, Y.-B., 2013. Robust control allocation design for marine vessel. Ocean Eng. 63, 105-111.

Khalil, H.K., 2002. Nonlinear Systems, 3rd edition. Prentice Hall, New York, NY, USA.

Morse, A.S., 1993. A gain matrix decomposition and some of its applications. Syst. Control Lett. 21 (1), 1-10.

Skjetne, R., Smogeli, O.N., Fossen, T.I., 2004. A nonlinear ship manoeuvering model: identification and adaptive control with experiments for a model ship. Model. Identif. Control 25 (1), 3-27.

Smith, E., Feemster, M., Esposito, J.M., 2007. Swarm manipulation of an unactuated surface vessel, in: Proceedings of Southeastern Symposium on System Theory, Macon, GA, USA, pp. 16-20.

Topp, J., Feemster, M., 2010. An adaptive control design for a system with unknown control direction: Experimental results, in: Proceedings of Southeastern Symposium on System Theory, Tyler, TX, USA, pp. 81-84.

Tran, V.L., Im, N., 2012. A study on ship automatic berthing with assistance of auxiliary devices. Int. J. Naval Archit. Ocean Eng. 4, 199-210.

Vlachos, K., Papadopoulos, E., 2013. Modeling and control of a novel over-actuated marine floating platform. Ocean Eng. 63, 10-22.

Xian, B., Dawson, D.M., de Queiroz, M.S., Chen, J., 2004. A continuous asymptotic tracking control strategy for uncertain nonlinear systems. IEEE Trans. Autom. Control 49 (7), 1206-1211. 